\title{
CS LINES AND DUST CONTINUUM OBSERVATIONS OF THE OMC2 INFRARED CLUSTER
}

\author{
T. UMEMOTO, N. OHASHI, Y. MURATA, K. TATEMATSU, \& \\ M. SUZUKI \\ Nobeyama Radio Observatory, National Astronomical Observatory, \\ Minamimaki, Minamisaku, Nagano 384-13, Japan
}

\section{Introduction}

It is known that stars in GMCs are often born as clusters. Recently, near infrared imaging has enabled us to study the young stars within molecular clouds (e.g., Lada \& Lada 1991). Orion Molecular Cloud 2 (OMC2) is located 12'. north of the Trapezium cluster in the Orion A cloud, and contains a cluster of about 20 near-IR sources and several FIR sources distributed within a diameter of 0.2 pc (Rayner et al.1989; Johnson et al. 1990; Mezger, Wink, \& Zylka 1990). By large scale mapping observations using the NRO $45 \mathrm{~m}$ telescope, this infrared cluster is found to be associated with a dense molecular core (Tatematsu et al.1993, Umemoto $e t$ al.1993). The region was observed using the Nobeyama Millimeter Array (NMA) to elucidate the structure and cluster formation process within a core.

\section{Results and Discussion}

Single- dish CS $(1-0)$ data show that the infrared cluster is associated with a dense core having a mass of $230 \mathrm{M}_{\odot}$ and a radius of $0.18 \mathrm{pc}$. Assuming the total stellar mass is $20 \mathrm{M}_{\odot}$, the star formation efficiency is estimated to be $8 \%$, which is lower than $22 \%$ in the pOph core (Wilking, Lada, \& Young 1989).

The interferometer observations of the CS(2-1) line and continuum emission at $98 \mathrm{GHz}$ show several features of emission corresponding to previously known sources. In paticular, we detected strong CS line and continuum emission toward the FIR4 detected in $\lambda 1.3 \mathrm{~mm}$ dust continuum. Continuum emission was also detected toward the luminous NIR source IRS4 (FIR3) and FIR 5 (Fig. 1). The mass of dust clumps was estimated to be $1.9 \mathrm{M}_{\odot}$ (FIR3), $3.3 \mathrm{M}_{\odot}$ (FIR4), and $0.76 \mathrm{M}_{\odot}$ (FIR 5 ), assuming the dust temperature was $40 \mathrm{~K}$. These values are a factor of 10 lager than T Tauri stars (Ohashi et al.1991). We cannot detect T Tauri stars in this region due to the high detection limit of these observations.

From velocity-channel maps, we found 7 small clumps of CS emission (Fig. 2). The mean radius of clumps was $0.02 \mathrm{pc}, 1 / 10$ of the molecular cloud core radius. The mass of clumps ranged from 0.38 to $12 \mathrm{M}_{\odot}$ (all $16 \mathrm{M}_{\odot}$ ) (assuming $T_{\mathrm{ex}}$ $=40 \mathrm{~K}$ ). These suggest that a substructure exists within the molecular cloud core. We also found clumps without NIR or FIR sources, which might evolve into the next generation of cluster members.

The high-velocity CS(2-1) emission was detected around FIR4, but the center of the emission does not coincide with FIR4. The high-velocity emission shows a bipolar pattern with east-west orientation and 23 !'separation from a previously known bipolar outflow source (Fisher et al.1985). This indicates that there is a new bipolar outflow source which does not have a strong continuum source. 
If the lower SFE is due to the early evolutionary stage of the cluster formation, the lower SFE together with recent star formation may indicate that the $\mathrm{OMC2}$ is now in the process of forming a cluster.

\section{References}

Fischer, J., Sanders, D. B., Simon, M., \& Solomon, M. 1985, ApJ, 293, 508 Johnson, J. J. et al.1990, AJ, 100, 518

Lada, C. J. \& Lada, E.A. 1991, in The Formation and Evolution of Star Clusters, A.S.P. Conference Series No.11, ed. K.A. James, (Chelsea: Michigan), p3 Mezger, P. G., Wink, J. E., \& Zylka, R. 1990, A\&A, 228, 95

Ohashi, N., Kawabe, R., Hayashi, M., Ishiguro, M. 1991, AJ, 102, 2054

Rayner, J., McLean, I., McCaughrean, M., \& Aspin, C. 1989, MNRAS, 241, 469 Tatematsu, K. et al.1993, ApJ, 404, 643

Umemoto, T. et al.1993 in preparation Wilking, B.A., Lada, C.J. \& Young, E.T. 1989, ApJ, 340, 823
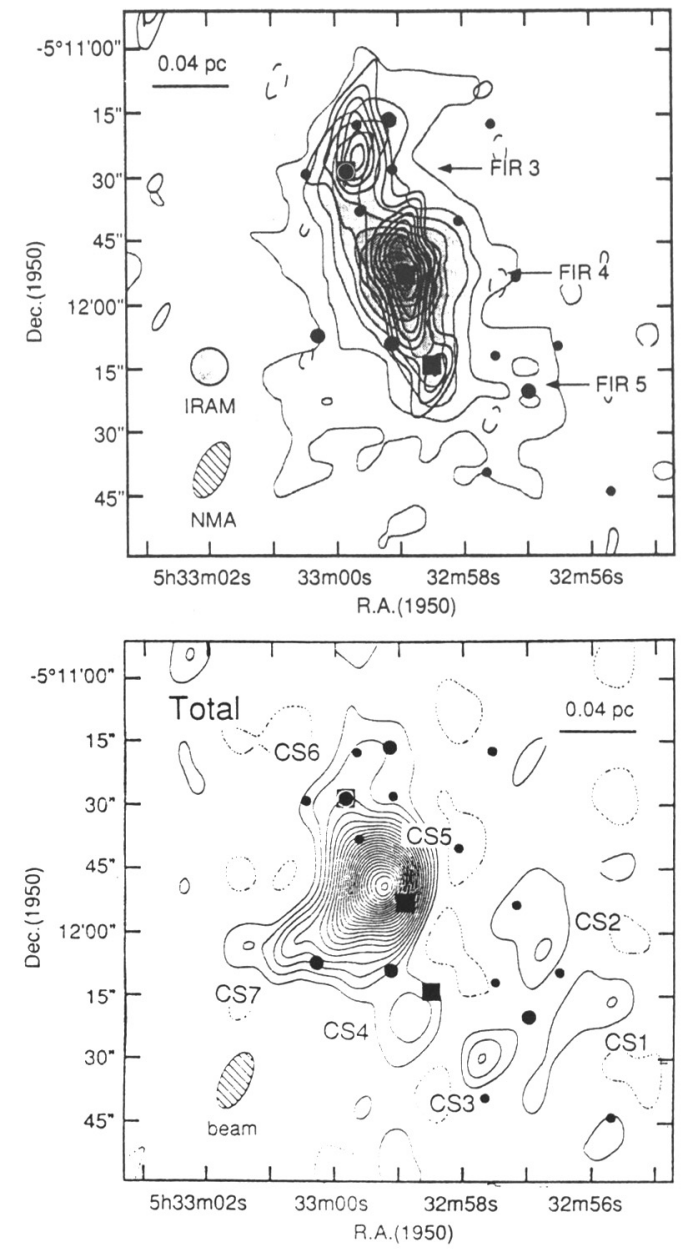

Figure 1. $\lambda 3 \mathrm{~mm}$ continuum map compared with $\lambda 1.3 \mathrm{~mm}$ continuum map obtained by Mezger, Wink, \& Zylka (1990). The lowest contour and contour interval for $\lambda 3 \mathrm{~mm}$ continuum map are $220 \mathrm{mJy}^{\circ}$ beam $^{-1}$ and 7.0 $\mathrm{mJy} \cdot$ beam $^{-1}$. The filled circles and filled squares represent NIR stars (Johnson et al. 1990 ; Rayner et al. 1989) and $\lambda 1.3 \mathrm{~mm}$ continuum sources (FIR3-FIR5).

Figure 2. Total intensity map of the CS (2-1) emission integrated over a velocity range from $3.8 \mathrm{~km}$ $\cdot \mathrm{s}^{-1}$ to $16.3 \mathrm{~km} \cdot \mathrm{s}^{-1}$. The lowest contour and contour interval are $2 \sigma$, where the $1 \sigma$ level is $830 \mathrm{mJy}$ - beam ${ }^{-1}$. CS1 - CS7 indicate the clumps of CS (2-1) emission. 